Pacific Journal of Mathematics

REGULARIZED DISTANCE AND ITS APPLICATIONS 


\title{
REGULARIZED DISTANCE AND ITS APPLICATIONS
}

\author{
GARY M. LIEBERMAN
}

\begin{abstract}
One of the most powerful tools in studying second order elliptic and parabolic differential equations is the barrier method, i.e. using the comparison principle with a suitable comparison or barrier function to infer some feature of the boundary behavior of a solution to such an equation. For sufficiently smooth domains $\Omega$ (e.g. $\partial \Omega \in C^{2}$ ), barrier functions can be constructed rather easily in terms of the distance function $d(x)=\operatorname{dist}(x, \partial \Omega)$ because $d$ is a $C^{2}$ function near $\partial \Omega$; for less smooth domains it need not be even $C^{1}$ (although it is Lipschitz continuous.) These less smooth domains are of interest and several authors have constructed barriers for certain such domains. We consider here a general method for constructing these barriers by introducing a regularized distance, described below.
\end{abstract}

Our concern here is primarily to develop a general theory of this regularized distance; we merely indicate some applications. We note that many of the ideas are not new and have appeared before in specialized circumstances. For example, essentially a regularized distance has been

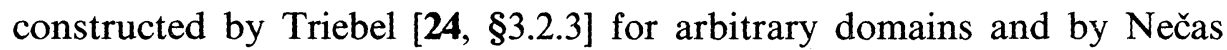
[22, Theorem 2.1] for Lipschitz domains. Both constructions are slightly different from the one given here. In addition we explore aspects of regularized distance not considered by Triebel or Nečas.

We mention also some authors who have used some of these additional properties of the regularized distance. Výborný used a regularized distance in [25] although he took the existence of such a function as the geometric characterization of his domains. Gilbarg and Hörmander constructed and used essentially a regularized distance for $C^{1+\alpha}$ domains in [3]. Finally Lieberman used a regularized distance both for $C^{1+\alpha}$ domains and for convex domains in [18]. Proofs of the properties asserted there are contained in the present work.

This work is organized as follows. We derive basic properties of the regularized distance, including its existence for arbitrary domains, in $§ 1$. A local regularized distance for Lipschitz domains is discussed in $\$ 2$, and a modified regularized distance especially suited for parabolic equations is constructed in $\S 3$. Some simple applications appear in $\$ 4$. 
1. Existence and basic properties of regularized distance. Let $\Omega$ be an open subset of $\mathbf{R}^{n}$ having non-empty boundary $\partial \Omega$. We define the signed distance to $\partial \Omega$ by

$$
d(x)=\left\{\begin{array}{rr}
\operatorname{dist}(x, \partial \Omega) & x \in \Omega \\
-\operatorname{dist}(x, \partial \Omega) & x \notin \Omega .
\end{array}\right.
$$

We call a function $\rho$ a regularized distance for $\Omega$ if $\rho \in C^{2}\left(\mathbf{R}^{2} \backslash \partial \Omega\right) \cap$ $\mathbf{C}^{0,1}\left(\mathbf{R}^{n}\right)$ and if the ratios $\rho(x) / d(x)$ and $d(x) / \rho(x)$ are positive and uniformly bounded for all $x \in \mathbf{R}^{n} \backslash \partial \Omega$. To construct a regularized distance, we use a modification of a standard mollification argument.

LEMMA 1.1. Let $\Omega$ be an open subset of $\mathbf{R}^{n}$ having non-empty boundary, and suppose there is a Lipschitz function $g$ for which the ratios $d / g$ and $g / d$ are uniformly bounded and positive in $\mathbf{R}^{n} \backslash \partial \Omega$. Let $L$ be a positive constant such that $|g(x)-g(y)| \leq \frac{1}{2} L|x-y|$ for all $x$ and $y$ in $\mathbf{R}^{n}$, let $\phi$ be a non-negative $C^{2}\left(\mathbf{R}^{n}\right)$ function with support in the unit ball such that $\int_{\mathbf{R}^{n}} \phi(z) d z=1$ and define

$$
G(x, \tau) \int_{|z|<1} g(x-(\tau / L) z) \phi(z) d z
$$

Then a regularized distance is given by the equation

$$
\rho(x)=G(x, \rho(x)) .
$$

Proof. To see that $\rho$ is a regularized distance, we first investigate the properties of $G$.

For $\tau \neq 0$, we can write

$$
G(x, \tau)=\left(-\frac{L}{\tau}\right)^{n} \int_{\mathbf{R}^{n}} g(z) \phi(L(x-z) / \tau) d z ;
$$

it is clear from this formula that $G \in C^{2}\left(\mathbf{R}^{n+1} \backslash\{(x, 0)\}\right)$ since $g$ is continuous and the integration is over a compact set. Moreover

$$
G\left(x, \tau_{1}\right)-\left(x, \tau_{2}\right)=\int_{|z|<1}\left[g\left(x-\left(\tau_{1} / L\right) z\right)-g\left(x-\left(\tau_{2} / L\right) z\right)\right] \phi(z) d z,
$$

so the choice of $L$ implies that

$$
\begin{aligned}
\left|G\left(x, \tau_{1}\right)-G\left(x, \tau_{2}\right)\right| & \leq \int_{|z|<1} \frac{1}{2} L\left|\tau_{1}-\tau_{2}\right|(|z| / L) \phi(z) d z \\
& \leq \frac{1}{2}\left|\tau_{1}-\tau_{2}\right| .
\end{aligned}
$$

Similarly

$$
\left|G\left(x_{1}, \tau\right)-G\left(x_{2}, \tau\right)\right| \leq \frac{1}{2} L\left|x_{1}-x_{2}\right| .
$$


We now consider (1.1.). By virtue of (1.3), (1.1) has a unique solution for every $x \in \mathbf{R}^{n}$, which can be found by iteration. If $\rho_{0}(x) \equiv 0$ and $\rho_{m}(x)=G\left(x, \rho_{m-1}(x)\right)$, then $\left|\rho_{m}(x)-\rho_{m-1}(x)\right| \leq \frac{1}{2}\left|\rho_{m-1}(x)-\rho_{m-2}(x)\right|$, so the infinite series $\sum_{m=1}^{\infty}\left(\rho_{m}(x)-\rho_{m-1}(x)\right)$ is uniformly and absolutely convergent, with sum $\rho(x)$ a solution of (1.1). The uniqueness of $\rho$ follows by noting that any two solutions $\rho_{1}$ and $\rho_{2}$ of (1.1) satisfy the inequality

$$
\left|\rho_{1}(x)-\rho_{2}(x)\right|=\left|G\left(x, \rho_{1}(x)\right)-G\left(x, \rho_{2}(x)\right)\right| \leq \frac{1}{2}\left|\rho_{1}(x)-\rho_{2}(x)\right| .
$$

Another application of (1.3) yields

$$
G(x, 0)-\frac{1}{2}|\rho(x)| \leq \rho(x) \leq G(x, 0)+\frac{1}{2}|\rho(x)| ;
$$

since $G(x, 0)=g(x)$, we see that $\frac{1}{2} \leq \rho(x) / g(x) \leq 2$ and hence that $\rho / d$ and $d / \rho$ are positive and uniformly bounded. Combining (1.3), (1.4) and the equation

$$
\begin{aligned}
\rho(x)-\rho(y)= & (G(x, \rho(x))-G(x, \rho(y))) \\
& +(G(x, \rho(y))-G(y, \rho(y)))
\end{aligned}
$$

yields

$$
|\rho(x)-\rho(y)| \leq L|x-y|,
$$

so $\rho$ is Lipschitz. That $\rho$ is $C^{2}$ follows from the implicit function theorem, and therefore $\rho$ is a regularized distance.

It follows from the proof of Theorem 1.3 below that in fact $\rho \in$ $C^{3}\left(\mathbf{R}^{n} \backslash \partial \Omega\right)$ and that $\left|D^{k} \rho\right|=O\left(|\rho|^{1-k}\right)$ for $k=0,1,2,3$ (cf. [15, Lemma 4.13]). In addition it is clear that higher regularity of $\rho$ can be obtained by increasing the regularity of $\phi$. In particular if $\phi \in C^{\infty}\left(\mathbf{R}^{n}\right)$, then $\rho \in$ $C^{\infty}\left(\mathbf{R}^{n} \backslash \partial \Omega\right)$ and $\left|D^{k} \rho\right|=O\left(|\rho|^{1-k}\right)$ for all non-negative integers $k$.

Lemma 1.1 reduces the study of regularized distance to choosing a suitable function $g$. We illustrate this procedure with various choices for $g$ relevant to the properties of $\Omega$ we wish to consider. First we choose $g \equiv d$ to obtain a result similar to that in $[\mathbf{2 4}, \S 3.2 .3]$.

COROLlaRY 1.2. Every domain has a regularized distance.

Proof. We need only verify that $d$ is Lipschitz and use $g=d$ in Lemma 1. We shall show that $|d(x)-d(y)| \leq|x-y|$. If $x$ and $y$ are both in $\Omega$ or if neither is in $\Omega$, we proceed as in $[4, \S 14.6]$. Let $z \in \partial \Omega$ be a point such that $|y-z|=|d(y)|$. Then

$$
|d(x)| \leq|x-z| \leq|x-y|+|d(y)| .
$$


Reversing the roles of $x$ and $y$, we obtain

$$
|d(x)-d(y)|=|| d(x)|-| d(y)|| \leq|x-y| .
$$

On the other hand, if $x \in \Omega$ and $y \notin \bar{\Omega}$, let $z \in \partial \Omega$ be a point on the line segment joining $x$ and $y$. Then

$$
|x-y|=|x-z|+|z-y| \geq|d(x)|+|d(y)|=|d(x)-d(y)| .
$$

Combining these two cases gives the desired result.

Although this result has some independent interest, there are certain desirable features (cf. $[4, \S 14.6]$ ) of the distance function for $C^{2}$ domains which do not seem to have an analog for the regularized distance constructed in this corollary. The two such features we shall use in the application are (1) that $|D \rho|$ be bounded away from zero near $\partial \Omega$, and (2) that geometric properties of $\Omega$ be represented by analytic properties of $\rho$. To make (1) precise, we say that a regularized distance is proper if there are positive constants $c_{1}$ and $c_{2}$ such that $|D \rho(x)| \geq c_{1}$ whenever $0<$ $|\rho(x)| \leq c_{2}$. With regard to (2), we consider both regularity and convexity. We shall describe regularity of $\partial \Omega$ in terms of the regularity of the function $g$ of Lemma 1. The connection with regularity in terms of a local representation of $\partial \Omega$ will be discussed in the next section.

THEOREM 1.3. Let $\Omega$ and $g$ be as in Lemma 1.1 and suppose that $g \in C^{1}\left(\mathbf{R}^{n}\right)$. Then $\Omega$ has a $C^{1}$ regularized distance. Let $\zeta$ be a continuous, increasing function with $\zeta(0)=0$. If

$$
|D g(x)-D g(y)| \leq \zeta(|x-y|) \text { for all } x, y \text { in } \mathbf{R}^{n}
$$

(so Dg is uniformly continuous), if L and $\phi$ are as in Lemma 1 , and if

$$
K=\int_{|z|<1}|D \phi(z)| d x
$$

then $\rho$ is proper and for $i, j=1, \ldots, n$ we have

$$
\begin{array}{r}
|D \rho(x)-D \rho(y)| \leq 8 \zeta(|x-y|) \quad \text { for all } x, y \text { in } \mathbf{R}^{n} \\
\left|D_{i j} \rho(x)\right| \leq 2(4 K+3 n+1)(L /|\rho(x)|) \zeta(|\rho(x)| / L) \\
\quad \text { for all } x \in \mathbf{R}^{n} \backslash \partial \Omega .
\end{array}
$$

Proof. First we note that $G$ is $C^{1}$ since $g$ is $C^{1}$ and use the implicit function theorem to see that $\rho$ is $C^{1}$.

Before proceeding further, we simply notation as follows. Subscripts on $G$ will denote partial derivatives, $\tau$ will be identified with $x_{n+1}$ and the 
argument $\rho(x)$ will be suppressed from $G$ and its derivatives. Thus

$$
G_{i}(x)=\frac{\partial G}{\partial x_{i}}(x, \rho(x)), \quad G_{n+1}(y)=\frac{\partial G}{\partial \tau}(y, \rho(y)), \quad \text { etc. }
$$

Also we denote by $G^{\prime}(x)$ the vector $\left(G_{1}(x), \ldots, G_{n}(x)\right)$.

Differentiating (1.1) yields

$$
\begin{aligned}
D \rho(x)-D \rho(y)= & \left(G^{\prime}(x)-G^{\prime}(y)\right)+D \rho(y)\left(G_{n+1}(x)-G_{n+1}(y)\right) \\
& +G_{n+1}(x)(D \rho(x)-D \rho(y)) .
\end{aligned}
$$

Combining this equation with (1.3) and (1.5), we see that

$$
|D \rho(x)-D \rho(y)| \leq 2\left|G^{\prime}(x)-G^{\prime}(y)\right|+2 L\left|G_{n+1}(x)-G_{n+1}(y)\right| \text {. }
$$

to obtain (1.7a) we estimate the two terms on the right side of (1.8).

Since $g \in C^{1}$, we can differentiate under the integral to obtain

$$
\begin{aligned}
G^{\prime}(x) & =\int_{|z|<1} D g(x-(\rho(x) / L) z) \phi(z) d z \\
G_{n+1}(x) & =-1 / L \int_{|z|<1} z \cdot D g(x-(\rho(x) / L) z) \phi(z) d z .
\end{aligned}
$$

Hence

$$
\begin{aligned}
\mid G^{\prime}(x) & -G^{\prime}(y) \mid \\
\leq & \int_{|z|<1}|D g(x-(\rho(x) / L) z)-D g(y-(\rho(x) / L) z)| \phi(z) d z \\
& +\int_{|z|<1}|D g(y-(\rho(x) / L) z)-D g(y-(\rho(y) / L) z)| \phi(z) d z \\
\leq & \int_{|z|<1}(\zeta(|x-y|)+\zeta(|\rho(x)-\rho(y)| / L)) \phi(z) d z \\
\leq & 2 \zeta(|x-y|) \quad \text { by }(1.5) .
\end{aligned}
$$

Similarly

$$
\left|G_{n+1}(x)-G_{n+1}(y)\right| \leq 2 \zeta(|x-y|) / L .
$$

Inserting these last two estimates in (1.8) gives (1.7a).

To derive (1.7b), we differentiate (1.1) twice to obtain

$$
\begin{aligned}
D_{i j} \rho(x)= & 1 /\left(1-G_{n+1}(x)\right) \\
& \cdot\left[G_{i j}(x)+G_{i, n+1}(x) D_{J} \rho(x)+G_{J, n+1}(x) D_{i} \rho(x)\right. \\
& \left.+G_{n+1, n+1}(x) D_{i} \rho(x) D, \rho(x)\right] .
\end{aligned}
$$


So to estimate $D_{\imath j} \rho$, we must evaluate and estimate the second derivatives of $G$. The evaluations are routine but tedious. We take the equations (1.9) with $\rho(x)$ replaced by $\tau$, make the substitution $y=x-(\tau / L) z$ (to obtain equations similar to (1.2)), differentiate, and then convert back to the integration variable $z$. The result is that

$$
\begin{aligned}
& \text { (1.11) } \quad G_{\imath \jmath}(x)=-(L / \rho(x)) \int_{|z|<1} D_{l} g(x-(\rho(x) / L) z) D_{j} \phi(z) d z \\
& G_{l, n+1}(x)=-(1 / \rho(x)) \int_{|z|<1} D_{l} g(x-(\rho(x) / L) z) d_{j}\left(z_{j} \phi(z)\right) d z \\
& G_{n+1, n+1}(x) \\
& \quad=-(1 /(L \rho(x))) \int_{|z|<1} D_{k} g(x-(\rho(x) / L) z) D_{j}\left(z_{k} z_{j} \phi(z)\right) d z .
\end{aligned}
$$

Since $\phi$ has compact support,

$$
\int_{|z|<1} D_{j} \phi(z) d z=0
$$

and hence

$$
G_{\imath \jmath}(x)=-(L / \rho(x)) \int_{|z|<1}\left[D_{i} g(x-(\rho(x) / L) z)-D_{\imath} g(x)\right] D_{j} \phi(z) d z
$$

with analogous expressions for $G_{i, n+1}(x)$ and $G_{n+1, n+1}(x)$. The integrals of these expressions are estimated by noting that

$$
\left|D_{1} g(x-(\rho(x) / L) z)-D_{i} g(x)\right| \leq \zeta(|\rho(x)| / L)
$$

so

$$
\begin{aligned}
& \left|G_{i j}(x)\right| \leq K L \zeta(|\rho(x)| / L) /|\rho(x)| \\
& \left|G_{l, n+1}(x)\right| \leq(n+K) \zeta(|\rho(x)| / L) /|\rho(x)| \\
& \left|G_{n+1, n+1}(x)\right| l \leq(n+1+K) \zeta(\mid \rho(x) / L) /(|\rho(x)| L) .
\end{aligned}
$$

Inequality $(1.7 \mathrm{~b})$ follows readily from these inequalities in conjunction with (1.3), (1.5), and (1.10).

By virtue of the continuity of $D \rho$, we infer that $\rho$ is proper provided $|D \rho|$ is bounded away from zero on $\partial \Omega$. Now if $x \in \partial \Omega$, then

$$
\begin{aligned}
|D \rho(x)| & =|D g(x)| /\left(1+D g(x) \cdot \int_{|z|<1} z \phi(z) d z\right) \\
& \geq 2|D g(x)| /(2+L)
\end{aligned}
$$


since $|D g| \leq \frac{1}{2} L$. Thus $\rho$ is proper if $|D g|$ is bounded away from zero on $\partial \Omega$, so let $x \in \partial \Omega$.

Suppose first that $\Omega$ satisfies an interior sphere condition at $x$ with center $x_{0}$, and let $M$ be a positive constant such that $g(y) \geq M d(y)$ for all $y \in \mathbf{R}^{n}$. If $\omega$ is the vector from $x$ to $x_{0}$, it is clear that

$$
d(x+\omega)=t|\omega| \text { for } 0 \leq t \leq 1,
$$

and hence

$$
g(x+\omega)-g(x)=g(x+t \omega) \geq M t|\omega| \text { for } 0 \leq t \leq 1 .
$$

Therefore $\omega \cdot D g(x) \geq M|\omega|$, so $|D g(x)| \geq M$.

To complete the proof, we need only show that the set of points of $\partial \Omega$ at which $\Omega$ satisfies an interior sphere condition is dense in $\partial \Omega$, so let $x \in \partial \Omega$ and $\delta>0$ be arbitrary, and choose $x_{1} \in \Omega$ and $x_{2} \in \partial \Omega$ such that

$$
\left|x-x_{1}\right|<\delta / 2, \quad\left|x_{1}-x_{2}\right|=d\left(x_{1}\right) \text {. }
$$

Since $d\left(x_{1}\right)<\delta / 2$, it follows that $\left|x-x_{2}\right|<\delta$; also $\Omega$ satisfies an interior sphere condition (with center $x_{1}$ and radius $d\left(x_{1}\right)$ ) at $x_{2}$. Thus the set of points of $\partial \Omega$ at which $\Omega$ satisfies an interior sphere condition is dense in $\partial \Omega$. From the continuity of $D g$ it follows that $|D g| \geq M$ on $\partial \Omega$ and hence $\rho$ is proper.

We remark that a regularized distance constructed with smoother $g$ or $\phi$ will obey an estimate on its higher derivatives analogous to (1.7). Moreover modulus of continuity estimates for these higher derivatives can be obtained in terms of the function $\zeta$ (cf. [3, Lemma 2.8] and Theorem 4.1 below) under the hypotheses of the preceding theorem. We remark also that the condition that $g / d$ and $d / g$ be uniformly bounded is equivalent, in this case, to $D g$ being bounded away from zero on $\partial \Omega$.

We now consider convex domains. For technical reasons (which will become clear later), we only consider bounded convex domains.

THEOREM 1.4. Let $\Omega$ be an open, bounded, convex subset of $\mathbf{R}^{n}$. Then $\Omega$ has a concave proper regularized distance $\rho$, i.e. the matrix $\left(D_{i j} \rho(x)\right)$ is negative semi-definite for $x \in \mathbf{R}^{n} \backslash \partial \Omega$.

Proof. Without loss of generality, we may assume that $0 \in \Omega$. Define the function $h(x)$ by

$$
h(x)=\inf \{\lambda>0: x \in \lambda \Omega\} .
$$

(This is the well-known Minkowski distance function. Although all of our assertions concerning $h$ can be derived from known results in the theory of 
convexity, e.g. from results on [17], we shall give elementary direct proofs.) We shall show that

$h$ is convex, i.e., $h(t x+(1-t) y) \leq t h(x)+(1-t) h(y)$ if $0<t<1$ $h$ is uniformly Lipschitz, $|x| /|h(x)|$ is uniformly bounded for $x \neq 0$.

To prove the convexity, suppose $x \in \lambda \Omega$ and $y \in \mu \Omega$, say $x=\lambda x_{1}$, $y=\mu y_{1}$. Then

$$
t x+(1-t) y=t \lambda x_{1}+(1-t) \mu y_{1} .
$$

Setting $\alpha=t \lambda /(t \lambda+(1-t) \mu)$ and noting that $0<\alpha<1$, we obtain

$$
t x+(1-t) y=(t \lambda+(1-t) \mu)\left[\alpha x_{1}+(1-\alpha) y_{1}\right] \text {. }
$$

Since $\Omega$ is convex, this equation implies that

$$
t x+(1-t) y \in(t \lambda+(1-t) \mu) \Omega
$$

so $h(t x+(1-t) y) \leq t \lambda+(1-t) \mu$, and hence $h$ is convex.

To prove the uniform Lipschitz continuity, set $r=2 / d(0)$, and suppose $x \in \lambda \Omega$, say $x=\lambda x_{1}$. Then

$$
y=\lambda x_{1}+y-x=\lambda x_{1}+[(1 / r)(y-x) /|y-x|] r|y-x| .
$$

Clearly $z=(1 / r)(y-x) /|y-x| \in \Omega$ since $|z|=1 / r \leq d(0) / 2$, i.e. $z$ is closer to 0 than $\partial \Omega$ is. Thus, setting $\alpha=\lambda /(\lambda+r|y-x|)$,

$$
y=\lambda x_{1}+r|y-x| z=(\lambda+r|y-x|)\left(\alpha x_{1}+(1-\alpha) z\right),
$$

so $h(y) \leq h(x)+r|y-x|$. Upon reversing the roles of $x$ and $y$, we obtain

$$
|h(x)-h(y)| \leq r|x-y| \text {. }
$$

To obtain a bound on $|x| /|h(x)|$, suppose $|y| \leq K$ for all $y \in \Omega$. Then for $x \neq 0$, we have

$$
|(1 / 2 K)| x|y| \leq|x| / 2+|x|
$$

for all $y \in \Omega$ and hence $x \notin(|x| / 2 K) \Omega$. Since $\Omega$ is convex and $0 \in \Omega$, it follows that $x \notin \lambda \Omega$ for all $\lambda \leq|x| / 2 K$. Hence $|h(x)| \geq|x| / 2 K$ for $x \neq 0$, so $|x| /|h(x)| \leq 2 K$.

Let us now define

$$
g(x)=1-h(x)
$$

and note that $g$ is uniformly Lipschitz since $h$ is. Since $g=0$ on $\partial \Omega$, it follows that if $|x-z|=d(x)$ and $z \in \partial \Omega$, then

$$
|g(x)|=|g(x)-g(z)| \leq r|x-z|=r|d(x)| .
$$


Also it is readily seen that $(1 / h(x)) x \in \partial \Omega$, so

$$
|d(x)| \leq|x \in(1 / h(x)) x|=(|x| /|h(x)|)|g(x)| \leq 2 K|g(x)| .
$$

Clearly $g>0$ in $\Omega$ and $g<0$ outside $\bar{\Omega}$, so the ratios $g / d$ and $d / g$ are positive and uniformly bounded. Therefore $g$ satisfies the hypotheses of Lemma 1. Let $G$ and $\rho$ be as in that lemma. Since $g$ is concave, $\phi$ is non-negative, and

$$
\begin{aligned}
G(t x & +(1-t) y, t \tau+(1-t) \sigma) \\
& =\int_{|z|<1} g(t[x-(\tau / L) z]+(1-t)[y-(\sigma / L) z]) \phi(x) d z,
\end{aligned}
$$

it follows that $G$ is jointly concave in the variables $x$ and $\tau$. We now define $\rho_{m}$ inductively by $\rho_{0} \equiv 0$ and

$$
\rho_{m}(x)=\frac{2}{3} G\left(x, \rho_{m-1}(x)\right)+\frac{1}{3} \rho_{m-1}(x),
$$

and observe that $\rho(x)=\lim _{m \rightarrow \infty} \rho_{m}(x)$. Now if $\rho_{m-1}$ is concave, then

$$
\begin{aligned}
\rho_{m}(t x+(1-t) y)= & \frac{2}{3} G\left(t x+(1-t) y, \rho_{m-1}(t x+(1-t) y)\right) \\
& +\frac{1}{3} \rho_{m-1}(t x+(1-t) y) \\
\geq & \frac{2}{3} G\left(t x+(1-t) y, t \rho_{m-1}(x)+(1-t) \rho_{m-1}(y)\right) \\
+ & \frac{1}{3}\left|\rho_{m-1}(t x+(1-t) y)-t \rho_{m-1}(x)-(1-t) \rho_{m-1}(y)\right| \\
& +\frac{1}{3} \rho_{m-1}(t x+(1-t) y) \\
= & \frac{2}{3} G\left(t x+(1-t) y, t \rho_{m-1}(x)+(1-t) \rho_{m-1}(y)\right) \\
& +\frac{1}{3}\left(t \rho_{m-1}(x)+(1-t) \rho_{m-1}(y)\right) .
\end{aligned}
$$

Since $\rho_{0}$ and $G$ are concave, sending $m \rightarrow \infty$ shows that $\rho$ is concave. The semi-definiteness of $\left(D_{i j} \rho\right)$ is proved by standard calculus arguments (see [1], Thm. 3.6).

To show that $\rho$ is proper, we observe that

$$
h(t x)=t h(x) \text { for all } t>0 \text { and all } x \in \mathbf{R}^{n},
$$

and hence that

$$
G(t x, t \tau)=(1-t)+t G(x, \tau) \text { for all } t>0 \text { and all }(x, \tau) \in \mathbf{R}^{n+1} \text {. }
$$

Differentiating this equation with respect to $t$, setting $t=1$ and $\tau=\rho(x)$ yields

$$
x^{i} G_{i}(x)+\rho(x) G_{n+1}(x)=-1+\rho(x) .
$$

This equation and the equation for $D \rho$ imply that

$$
x \cdot D \rho(x)=\rho(x)+\left(1 /\left(G_{n+1}(x)-1\right)\right) .
$$


If $0<|\rho(x)| \leq 1 / 3$, it follows from this equation and (1.3) that

$$
|D \rho(x)| \geq 1 /(3|x|) .
$$

Since $|d(x)| \geq|\rho(x)| / 2 r$ and $|x| \leq K$, we conclude that $\rho$ is proper.

We remark that the regularized distance constructed in this theorem is as regular as the domain (in the sense used in Theorem 1.3). To see this, let $g_{1}$ be a function with the properties described in Theorem 1.3 on a bounded convex domain $\Omega$. Then $h(x)$ is defined implicitly by the equation $g_{1}(x / h(x))=0$. But

$$
\frac{d}{d t}\left(g_{1}(x / t)\right)=-x \cdot D g_{1}(x / t) / t^{2}
$$

and it is easy to check that this is non-zero in a neighborhood of $\partial \Omega$. Hence $h$ has the same regularity as $g_{1}$ near $\partial \Omega$, and therefore so does $\rho$. Of course away from $\partial \Omega, \rho$ is $C^{2}$. This observation will not be used in the applications.

We fruther remark that it can be shown that the regularized distance from the proof of Corollary 1.2 is concave if $\Omega$ is convex; however it is not apparent that this regularized distance is proper.

2. Local regularized distance. From the construction of regularized distance in $\S 1$, it is clear that local properties of $\rho$ are determined by local properties of $g$. In this section we explore some of these local properties and their relationship with properties of $\Omega$. Everything we wish to consider can be described via a local representation of $\partial \Omega$, defined below. We remark that our basic construction parallels that of [22, Theorem 2.1] (see also [15, §4]) although the idea of a proper regularized distance does not appear there.

To be specific, let $x_{0} \in \partial \Omega$, let $r>0$, and define

$$
B_{r}=\left\{x \in \mathbf{R}^{n}:\left|x-x_{0}\right|<r\right\}, \quad \Omega_{r}=B_{r} \cap \Omega .
$$

If there is a constant $r>0$ and a function $\rho \in C^{2}\left(B_{r} \backslash \partial \Omega\right)$ which is Lipschitz continuous on $\bar{B}_{r}$ and such that the ratios $d / \rho$ and $\rho / d$ are bounded and positive on $B_{r} \backslash \partial \Omega$, we call $\rho$ a local regularized distance at $x_{0}$. If there are positive constants $\delta$ and $A$, an orthonormal coordinate system $Y=\left(y^{\prime}, y^{n}\right)=\left(y^{1}, \ldots, y^{n-1}, y^{n}\right)$ with origin at $x_{0}$, and a function $f$ such that

$$
\Omega_{4 \delta}=\left\{y \in B_{4 \delta}: y^{n}>f\left(y^{\prime}\right)\right\}
$$

and

$$
\left|f\left(y_{1}^{\prime}\right)-f\left(y_{2}^{\prime}\right)\right| \leq A\left|y_{1}^{\prime}-y_{2}^{\prime}\right| \text { for } y_{1}^{\prime}, y_{2}^{\prime} \text { in } B_{4 \delta},
$$


we call $f$ a local representation for $\partial \Omega$ at $x_{0}$. We note that not every domain has a local representation at every point of its boundary.

For a fixed $x_{0} \in \partial \Omega$ at which a local representation $f$ exists, it is easy to verify that $g(y)=y^{n}-f\left(y^{\prime}\right)$ obeys the hypotheses of Lemma 1.1 with $\mathbf{R}^{n}$ replaced by $B_{2 \delta}$. Clearly $g$ is Lipschitz in $B_{2 \delta}$ with

$$
|g(x)-g(y)| \leq \max \{1, A\}|x-y| \text { for all } x, y \text { in } B_{2 \delta},
$$

and the ratios $d / g$ and $g / d$ are positive in $B_{2 \delta}$ with

$$
|d(x)| \leq|g(x)| \leq\left(A^{2}+1\right)^{1 / 2}|d(x)| \text { for } x \in B_{2 \delta} .
$$

If we choose $L=2 \max \{1, A\}$, the construction of $\rho$ in Lemma 1.1 can be carried out for $x \in B_{\delta}$. To see this, we observe first that $G(x, \tau)$ is defined for $x \in B_{\delta}$ and $|\tau| / L \leq \delta$. Since $f(0)=0$, it follows from (1.4) and the definition of $g$ that $|G(x, \tau)| \leq \frac{1}{2} L|x|+\frac{1}{2} \tau$. Hence $G(x, \cdot)$ is a self-map of the interval $\left[-L \delta, L \delta\right.$ ] if $x \in B_{\delta}$, so the construction of $\rho$ in Lemma 1.1 can be carried out in this case. Moreover $|\rho| / L<\delta$. Finally $\rho$ is proper because $g(x+\varepsilon \omega)-g(x)=\varepsilon$ for $\omega$ a unit vector in the $y^{n}$-direction, so $G_{n}(x) \equiv 1$ in $B_{\delta}$. In fact it is clear that $|D \rho| \geq 1 / 2$ in $B_{\delta}$.

When additional hypotheses are placed on $f$, they imply additional properties for $\rho$.

THEOREM 2.1. Let $x_{0} \in \partial \Omega$. If $\partial \Omega$ has a $C^{1}$ local representation $f$ at $x_{0}$, then $\Omega$ has a $C^{1}$ local regularized distance $\rho$ at $x_{0}$. Specifically if $\zeta$ is a increasing function such that $\zeta(0)=0$ and if

$$
\left|D f\left(x^{\prime}\right)-D f\left(y^{\prime}\right)\right| \leq \zeta(|x-y|) \text { for }\left|x^{\prime}\right|,\left|y^{\prime}\right|<4 \delta,
$$

then there is a positive constant $C_{1}$, determined only by $A, \delta$, and $n$, such that

$$
\begin{gathered}
|D \rho(x)-D \rho(y)| \leq 10 \zeta(|x-y|) \quad \text { for } x, y \text { in } B_{\delta} \\
\left|D^{2} \rho(x)\right| \leq C_{1} \zeta(|\rho(x)| / 2 \max \{1, A\}) /|\rho(x)| \text { for } x \in B_{\delta} .
\end{gathered}
$$

We say that $\Omega$ is convex at $x_{0} \in \partial \Omega$ if $\Omega_{r}$ is convex for some $r>0$. When $\Omega$ is convex at $x_{0}$, a elementary calculation (cf. [1, Proposition 3.4] shows that $\partial \Omega$ has a convex local representation at $x_{0}$. From this fact, we can proceed as in the last half of the proof of Theorem 1.4 to infer the next theorem.

THEOREM 2.2. Let $x_{0} \in \partial \Omega$ be a point at which $\Omega$ is convex. Then $\Omega$ has a concave local regularized distance at $x_{0}$.

Finally we show that the two senses of regularity (that of Theorem 2.1 and that of Theorem 1.3) are essentially equivalent. 
THEOREM 2.3. Let $\Omega$ be an open set in $\mathbf{R}^{n}$. If there is a function $g \in C^{1}\left(\mathbf{R}^{n}\right)$ such that the hypotheses of Lemma 1.1 are satisfied, then there is a local represesntation for $\partial \Omega$ at each point of $\partial \Omega$. If there is a local representation for $\partial \Omega$ at each point of $\partial \Omega$ and if the constants $A$ and $\delta$ in the definition of local representation can be chosen independent of the particular point $x_{0}$, then there is a function $g \in C^{1}\left(\mathbf{R}^{n}\right)$ which satisfies the hypotheses of Lemma 1.1.

Proof. The first implication is a simple consequence of the implicit function theorem. The second implication follows by a construction of the function $g$.

Let $\left\{B_{i}\right\}$ be a sequence of open balls of radius $\delta$ such that $\bigcup_{i=1}^{\infty} B_{l}=\mathbf{R}^{n}$ and such that there is a positive integer $N$ such that each point in $\mathbf{R}^{n}$ lies in at most $N$ of these balls. (For example, the $B_{l}$ 's could be centered at all points whose coordinates are integer multiplies of $\delta / n$ ). If $B_{i} \cap \partial \Omega$ is non-empty, then let $\rho_{l}$ be a $C^{1}$ regularized distance defined in $B_{l}$ and if $B_{i} \cap \partial \Omega$ is empty let $\rho_{i}$ be the regularized distance for $\Omega$ constructed in Corollary 1.2 . Let $\left(\eta_{i}\right)$ be a $C^{2}$ partition of unit subordinate to $\left(B_{i}\right)$ with $\sup _{\iota} \sup _{B_{l}}\left|D \eta_{i}\right|$ finite and define

$$
g(x)=\sum_{i=1}^{\infty} \eta_{i}(x) \rho_{i}(x) .
$$

It is clear that $g \in C^{1}\left(\mathbf{R}^{N}\right)$ and that $g$ is uniformly Lipschitz on $\mathbf{R}^{n}$. Since there are positive constants $c_{1}$ and $c_{2}$ such that

$$
c_{1}|d(x)| \leq\left|\rho_{i}(x)\right| \leq c_{2}|d(x)| \text { for } i=1,2, \ldots \text { and } x \in \mathbf{R}^{n},
$$

it follows that $g$ is the required function.

Of course detailed information about the modulus of continuity of the function $g$ can be obtained from a knowledge of the moduli of continuity of the local representations and vice versa. A closer examination of certain aspects of the regularity of domains, especially comparison of different definitions, can be found in [2].

3. Anisotropic regularized distance. In certain circumstances, e.g., for parabolic equations, the independent variables are not all treated equally. Rather than discuss this situation in its fullest generality, we shall work here only with an anisotropic regularized distance suitable for parabolic equations.

To conform with normal usage, we change our notation slightly. We consider open sets $Q$ in $\mathbf{R}^{n+1}$ and label points in $\mathbf{R}^{n+1}$ by $(x, t)=$ $\left(x^{1}, \ldots, x^{n}, t\right)$. When $g$ is defined on a subset of $\mathbf{R}^{n}$, we set

$$
D g=\left(D_{1} g, \ldots, D_{n} g\right), \quad g_{t}=D_{n+1} g \text {, etc. }
$$


provided the derivatives exist. For any open set $Q \subset \mathbf{R}^{n+1}$ and $t \in \mathbf{R}$, we define

$$
Q(t)=\left\{x \in \mathbf{R}^{n}:(x, t) \in Q\right\}, \quad I=\{t \in \mathbf{R}: Q(t) \neq \varnothing\} .
$$

For $(x, t) \in \mathbf{R}^{n}$ let $d_{p}(x, t)$ be the signed distance from $(x, t)$ to $\partial Q(t)$, and for $\left(x_{0}, t_{0}\right) \in \mathbf{R}^{n+1}$ and $r>0$, we define

$$
C_{r}=\left\{(x, t) \in \mathbf{R}^{n+1}:\left|x-x_{n}\right|<r,\left|t-t_{0}\right|<r^{2}\right\}, \quad Q_{r}=C_{r} \cap Q .
$$

By a regularized parabolic distance, we mean a function $\rho$ such that

(i) $D \rho, D^{2} \rho$, and $\rho_{t}$ exist and are continuous on $\mathbf{R}^{n} \times I \backslash \partial Q$,

(ii) there is a constant $C$ such that

$$
\left|\rho\left(x_{1}, t_{2}\right)-\rho\left(x_{2}, t_{2}\right)\right| \leq C\left(\left|x_{1}-x_{2}\right|+\left|t_{1}-t_{2}\right|^{1 / 2}\right),
$$

(iii) the ratios $\rho / d_{p}$ and $d_{p} / \rho$ are bounded and continuous on $\mathbf{R}^{n} \times I \backslash \partial Q$. Let $\left(x_{0}, t_{0}\right) \in \partial Q$. If there is a positive constant $r$ with $\left[t_{0}-r, t_{0}+r\right] \subset I$ and a function $\rho$ satisfying the definition of regularized parabolic distance with $\mathbf{R}^{n} \times I$ replaced by $C_{r}$, we call $\rho$ a local regularized parabolic distance at $\left(x_{0}, t_{0}\right)$.

For simplicity we consider only local regularized parabolic distances, which we call regularized distances in $C_{r}$ for brevity.

If there are constants $\delta$ and $A$, an orthonormal coordinate system $Y=\left(y^{\prime}, y^{n}\right)$ with origin at $x_{0}$ and a function $f$ such that

$$
Q_{4 \delta}=\left\{(y, t) \in C_{4 \delta}: y^{n}>f\left(y^{\prime}, t\right)\right\}
$$

and

$$
\begin{aligned}
& \left|f\left(y_{1}^{\prime}, t_{1}\right)-f\left(y_{2}^{\prime}, t_{2}\right)\right| \leq A\left(\left|y_{1}^{\prime}-y_{2}^{\prime}\right|+\left|t_{1}-t_{2}\right|^{1 / 2}\right) \\
& \qquad \text { for }\left|y_{1}^{\prime}\right|,\left|y_{2}^{\prime}\right|,\left|t_{1}\right|^{1 / 2},\left|t_{2}\right|^{1 / 2} \leq 4 \delta,
\end{aligned}
$$

we call $f$ a local representation for $\partial Q$ at $\left(x_{0}, t_{0}\right)$. We note that if $f$ is a local representation for $\partial Q$ at $\left(x_{0}, t_{0}\right)$, then $\left(x_{0}, t_{0}\right)$ must lie on the lateral surface of $\partial Q$ (see [11] for the definition).

From this $f$ we can construct a regularized distance in $C_{\delta}$. We let $\phi$ be a non-negative $C^{2}\left(\mathbf{R}^{n-1}\right)$ function with support in $\{|z|<1\}$ such that $\int_{|z|<1} \phi(z) d z=1$, we let $\eta$ be a non-negative $C^{2}(\mathbf{R})$ function with support in $(-1,0)$ with $\int_{-1}^{0} \eta(s) d s=1$, and we set

$$
K=\int_{-1}^{0}\left|\eta^{\prime}(s)\right| d s+\int_{|z|<1}|D \phi(z)| d z+\int_{-1}^{0}\left|\eta^{\prime \prime}(s)\right| d s
$$

note that $K \geq 2$. Setting $L=4\left(A^{2}+1\right)^{1 / 2}$ and

$$
\begin{aligned}
& F(y, t, \tau, \boldsymbol{\sigma}) \\
& \quad=y^{n}-\int_{-1}^{0} \int_{|z|<1} f\left(y^{\prime}-(\tau / L) z, t-\left(\sigma / K^{2} L^{2}\right) s\right) \phi(z) d z \eta(s) d s,
\end{aligned}
$$


for $(y, t) \in C_{\delta},|\tau|<L \delta,|\sigma|<L^{2} \delta^{2}$, it is readily verified that

$$
\begin{gathered}
\left|D_{i} F(y, t, \tau, \sigma)\right| \leq L / 4, \quad\left|D_{\sigma} F(y, t, \tau, \sigma)\right| \leq 1 /\left(4 \sigma^{1 / 2}\right), \\
\left|D_{\tau} F(y, t, \tau, \sigma)\right| \leq 1 / 4 .
\end{gathered}
$$

If we set $G(y, t, \tau)=F\left(y, t, \tau, \tau^{2} / 2\right)$, it follows that a regularized distance in $C_{\delta}$ is given implicitly by the equation

$$
\rho(y, t)=G(y, t, \rho(y, t)) .
$$

Although convexity is not of particular concern for such equations, we desire a parabolic analog of the condition $f \in C^{1}\left(B_{4 \delta}\right)$. To describe the analog, consider an increasing, continuous function $\zeta$ such that $\zeta(\cdot) / \cdot$ is decreasing. We then assume of $f$ that

$$
\begin{gathered}
\left|D f\left(y_{1}^{\prime}, t_{1}\right)-D f\left(y_{2}^{\prime}, t_{2}\right)\right| \leq \zeta\left(\left|y_{1}^{\prime}-y_{2}^{\prime}\right|\right)+\zeta\left(\left|t_{1}-t_{2}\right|^{1 / 2}\right) \\
\left|f\left(y, t_{1}\right)-f\left(y, t_{2}\right)\right| \leq\left|t_{1}-t_{2}\right|^{1 / 2} \zeta\left(\left|t_{1}-t_{2}\right|^{1 / 2}\right) .
\end{gathered}
$$

(When $\zeta(s)=C s^{\alpha}$ for constants $\alpha<1$ and $C$, this definition says that $f \in H^{1+\alpha, 1 / 2+\alpha / 2}$ in the sense of [16] and that $\partial Q \cap C_{4 \delta}$ is a surface of class $\Lambda_{1, \alpha, \alpha / 2}^{0,1,(1+\alpha) / 2}$ in the sense of [7].) from these inequalities we can infer estimates on $D \rho, D^{2} \rho$, and $\rho_{t}$ analogous to (1.7).

THEOREM 3.1. Let $Q$ be an open set on $\mathbf{R}^{n+1}$, let $\left(x_{0}, t_{0}\right) \in \partial Q$, and suppose $\partial Q$ has a local representation $f$ at $\left(x_{0}, t_{0}\right)$ for which (3.2) holds. Then there is a positive constant, $C=C(K, L, n)$ such that the regularized distance $\rho$ constructed above obeys the estimates

$$
\begin{gathered}
\left|D \rho\left(x_{1}, t_{1}\right)-D \rho\left(x_{2}, t_{2}\right)\right| \leq C\left[\zeta\left(\left|x_{1}-x_{2}\right|\right)+\zeta\left(\left|t_{1}-t_{2}\right|^{1 / 2}\right)\right] \\
\left|D^{2} \rho(x, t)\right| \leq C \zeta(|\rho(x, t)| / L) /|\rho(x, t)| \\
\left|\rho_{t}(x, t)\right| \leq C \zeta(|\rho(x, t)| / L) /|\rho(x, t)| .
\end{gathered}
$$

Proof. To verify (3.3a), we proceed as in Theorem 1.3 making judicious use of $(3.2 \mathrm{a}, \mathrm{b})$. For brevity, we write $\rho_{1}=\rho\left(x_{1}, t_{1}\right), \rho_{2}=\rho\left(x_{2}, t_{2}\right)$, and $f(j, k, m, r)=f\left(x_{j}^{\prime}-\left(\rho_{k} / L\right) z, t_{m}-\left(\rho_{r}^{2} / 2 K^{2} L^{2}\right) s\right)$ and similarly for derivatives of $f$. Also we assume, by relabelling points if necessary, that $\left|\rho_{1}\right| \geq\left|\rho_{2}\right|$.

An examination of the proof of Theorem 1.3 and the fact that $G_{\tau}=F_{\tau}+\tau F_{\sigma}$ show that we need only estimate

$$
A_{1}=D F\left(x_{1}, t_{1}, \rho_{1}, \rho_{1}^{2} / 2\right)-D F\left(x_{1}, t_{1}, \rho_{1}, \rho_{2}^{2} / 2\right)
$$

and

$$
B_{1}=\rho_{1} F_{\sigma}\left(x_{1}, t_{1}, \rho_{1}, \rho_{1}^{2} / 2\right)-\rho_{2} F_{\sigma}\left(x_{2}, t_{2}, \rho_{2}, \rho_{2}^{2} / 2\right) .
$$


To estimate $A_{1}$, we examine the two cases $\left(\left|\rho_{1}^{2}-\rho_{2}^{2}\right| / 2 K^{2} L^{2}\right)^{1 / 2} \leq$ $\left|\rho_{1}-\rho_{2}\right| / 2 L$ and $\left(\left|\rho_{1}^{2}-\rho_{2}^{2}\right| / 2 K^{2} L^{2}\right)^{1 / 2}>\left|\rho_{1}-\rho_{2}\right| / 2 L$. In the first case, we write

$$
A_{1}=\int_{-1}^{0} \int_{|z|<1}[D f(1,1,1,1)-D f(1,1,1,2)] \phi(z) d z \eta(s) d s
$$

and use (3.2a) and the monontonicity of $\zeta$ to infer that

$$
\left|A_{1}\right| \leq \zeta\left(\left|\rho_{1}-\rho_{2}\right| / 2 L\right) .
$$

In the second case, we write

$$
A_{1}=\left(-L / \rho_{1}\right) \int_{-1}^{0}[f(1,1,1,1)-f(1,1,1,2)] D \phi(z) d z \eta(s) d s
$$

and use $(3.2 \mathrm{~b})$ and the monotonicity of $\zeta(\cdot) / \cdot$ to infer that

$$
\left|A_{1}\right| \leq\left|\rho_{1}+\rho_{2}\right| \zeta\left(\left|\rho_{1}-\rho_{2}\right| / 2 L\right) / K\left|\rho_{1}\right| \text {. }
$$

Since $K \geq 2,\left|\rho_{1}\right| \geq\left|\rho_{2}\right|$, and $\left|\rho_{1}-\rho_{2}\right| \leq 2 L \max \left\{\left|x_{1}-x_{2}\right|,\left|t_{1}-t_{2}\right|^{1 / 2}\right\}$, it follows that in either case

$$
\left|A_{1}\right| \leq \zeta\left(\left|x_{1}-x_{2}\right|\right)+\zeta\left(\left|t_{1}-t_{2}\right|^{1 / 2}\right)
$$

To estimate $B_{1}$, we observe that

$$
\begin{aligned}
B_{1}= & \rho_{1}\left[F_{\sigma}\left(x_{1}, t_{1}, \rho_{1}, \rho_{1}^{2} / 2\right)-F_{\sigma}\left(x_{1}, t_{1}, \rho_{1}, \rho_{1}^{2} / 2\right)\right] \\
& +\rho_{1}\left[F_{\sigma}\left(x_{2}, t_{1}, \rho_{1}, \rho_{1}^{2} / 2\right)-F_{\sigma}\left(x_{2}, t_{2}, \rho_{1}, \rho_{1}^{2} / 2\right)\right] \\
& +\left[\rho_{1} F_{\sigma}\left(x_{2}, t_{2}, \rho_{1}, \rho_{1}^{2} / 2\right)-\rho_{2} F_{\sigma}\left(x_{2}, t_{2}, \rho_{2}, \rho_{1}^{2} / 2\right)\right] \\
& +\rho_{2}\left[F_{\sigma}\left(x_{2}, t_{2}, \rho_{2}, \rho_{1}^{2} / 2\right)-F_{\sigma}\left(x_{2}, t_{2}, \rho_{2}, \rho_{2}^{2} / 2\right)\right],
\end{aligned}
$$

and denote the terms in square brackets by $B_{2}, B_{3}, B_{4}, B_{5}$. For $B_{2}$ the two cases are $\left|x_{1}-x_{2}\right| \leq\left|\rho_{1}\right| / 2^{1 / 2} K L$ and $\left|x_{1}-x_{2}\right|>\left|\rho_{1}\right| / 2^{1 / 2} K L$. In the first case we write

$$
\begin{array}{r}
B_{2}=\left(1 / \rho_{1}^{2}\right) \int_{-1}^{0} \int_{|z|<1}[f(1,1,1,1)-f(2,1,1,1)] \phi(z) d z \\
\times\left(\eta(s)+s \eta^{\prime}(s)\right) d s
\end{array}
$$

and we observe that

$$
\int_{-1}^{0}\left(\eta(s)+s \eta^{\prime}(s)\right) d s=0
$$

and that

$$
\begin{aligned}
& f(1,1,1,1)-f(2,1,1,1) \\
& \begin{aligned}
=\left(x_{1}-x_{2}\right) \cdot \int_{0}^{1} D f\left(\lambda x_{1}+(1-\lambda)\right. & x_{2}-\left(\rho_{1} / L\right) z, t_{1} \\
& \left.-\left(\rho_{1}^{2} / 2 K^{2} L^{2}\right) s\right) d \lambda .
\end{aligned}
\end{aligned}
$$


Thus, writing $\bar{x}=\lambda x_{1}+(1-\lambda) x_{2}-\left(\rho_{1} / L\right) z, \bar{\eta}(s)=\eta(s)+s \eta^{\prime}(s)$, we have

$$
\begin{aligned}
B_{2}=\int_{-1}^{0} \int_{|z|<1} \int_{0}^{1}\left\{D f\left(\bar{x}, t_{1}-\left(\rho_{1}^{2} / 2 K^{2} L^{2}\right) s\right)-\right. & \left.D f\left(\bar{x}, t_{1}\right)\right\} d \lambda \\
& \times \phi(z) d z \bar{\eta}(s) d s
\end{aligned}
$$

and hence, using (3.2a) and the monotonicity of $\zeta(\cdot) / \cdot$,

$$
\begin{aligned}
B_{2} & \leq(1+K)\left|x_{1}-x_{2}\right| \zeta\left(\left|\rho_{1}\right| / 2^{1 / 2} K L\right) /\left|\rho_{1}\right|^{2} \\
& \leq(1+K) \zeta\left(\left|x_{1}-x_{2}\right|\right) / 2^{1 / 2} K L\left|\rho_{1}\right| .
\end{aligned}
$$

In the second case we observe that

$$
\begin{aligned}
f(j, 1,1,1)= & \left(-\rho_{1} / L\right) \int_{0}^{1} z \cdot D f\left(x_{J}-\left(\lambda \rho_{1} / L\right) z, t_{1}-\left(\rho_{1}^{2} / 2 K^{2} L^{2}\right) s\right) d \lambda \\
& +f\left(x_{J}, t_{1}-\left(\rho_{1}^{2} / 2 K^{2} L^{2}\right) s\right) .
\end{aligned}
$$

If we write $\bar{x}_{j}=x_{j}-\left(\lambda \rho_{1} / L\right) s$ and $\bar{t}=t_{1}-\left(\rho_{1}^{2} / 2 K^{2} L^{2}\right) s$, define $\bar{\eta}$ as before, and use (3.4), we see that

$$
\begin{aligned}
B_{2}=\left(1 / \rho_{1}^{2}\right)[- & \left(\rho_{1} / L\right) \int_{-i}^{0} \int_{|z|<1} \int_{0}^{1} z \cdot\left\{D f\left(\bar{x}_{1}, \bar{t}\right)-D f\left(\bar{x}_{2}, \bar{t}\right)\right\} d \lambda \\
& \times \phi(z) \bar{\eta}(s) d s \\
& \left.+\sum_{j=1}^{2}(-1)^{j} \int_{-1}^{0} \int_{|z|<1}\left\{f\left(x_{j}, \bar{t}\right)-f\left(x_{j}, t_{1}\right)\right\} \phi(z) d z \bar{\eta}(s) d s\right]
\end{aligned}
$$

and therefore, using (3.2) and the monotonicity of $\zeta$

$$
\begin{aligned}
\left|B_{2}\right| & \leq(1+K)\left[\zeta\left(\left|x_{1}-x_{2}\right|\right) / L\left|\rho_{1}\right|+2 \zeta\left(\left|\rho_{1}\right| / 2^{1 / 2} K L\right) / 2^{1 / 2} K L\left|\rho_{1}\right|\right] \\
& \leq 2(1+K) \zeta\left(\left|x_{1}-x_{2}\right|\right) / L\left|\rho_{1}\right| .
\end{aligned}
$$

In either case this inequality is valid for $B_{2}$.

To estimate

$$
\begin{aligned}
B_{3}=\left(1 / \rho_{1}^{2}\right) \int_{-1}^{0} \int_{|z|<1}[f(2,1,1,1)-f(2,1,2,1)] \phi(z) d z & \\
& \times\left(\eta(s)+s \eta^{\prime}(s)\right) d s,
\end{aligned}
$$

we consider the cases $\left|t_{1}-t_{2}\right| \leq \rho_{1}^{2}$ and $\left|t_{1}-t_{2}\right|>\rho_{1}^{2}$. In the first case, we use the inequality

$$
|f(2,1,1,1)-f(2,1,2,1)| \leq\left|t_{1}-t_{2}\right|^{1 / 2} \zeta\left(\left|t_{1}-t_{2}\right|^{1 / 2}\right) \leq\left|\rho_{1}\right| \zeta\left(\left|t_{1}-t_{2}\right|^{1 / 2}\right) .
$$


In the second case, we use (3.4) to infer that

$$
\begin{aligned}
B_{3}= & \left(1 / \rho_{1}^{2}\right) \int_{-1}^{0} \int_{|z|<1}\left(\left[f(2,1,1,1)-f\left(x_{2}-\left(\rho_{1} / L\right) z, t_{1}\right)\right]\right. \\
& \left.+\left[f\left(x_{2}-\left(\rho_{1} / L\right) z, t_{2}\right)-f(2,1,2,1)\right]\right) \phi(z) d z\left(\eta(s)+s \eta^{\prime}(s)\right) d s,
\end{aligned}
$$

in which case

$$
\begin{aligned}
\left|B_{3}\right| & \leq\left(2 / \rho_{1}^{2}\right)\left(\left|\rho_{1}\right| / 2^{1 / 2} K L\right) \zeta\left(\left|\rho_{1}\right| / 2^{1 / 2} K L\right) \\
& \leq \zeta\left(\left|\rho_{1}\right|\right) /\left|\rho_{1}\right| \leq \zeta\left(\left|t_{1}-t_{2}\right|^{1 / 2}\right) /\left|\rho_{1}\right|
\end{aligned}
$$

In either case, $\left|B_{3}\right| \leq(1+K) \zeta\left(\left|t_{1}-t_{2}\right|^{1 / 2}\right)\left|\rho_{1}\right|$.

To estimate $B_{4}$, we see that

$$
\begin{array}{r}
B_{4}=\int_{-1}^{0} \int_{|z|<1}\left[f(2,2,1,1) / \rho_{1}-f(2,2,2,1) / \rho_{2}\right] \phi(z) d z \\
\times\left(\eta(s)+s \eta^{\prime}(s)\right) d s
\end{array}
$$

by observing that

$$
\begin{aligned}
f(2, j, 2,1)= & -\left(\rho_{1} / L\right) \int_{0}^{1} z \cdot D f\left(x_{2}-\lambda\left(\rho_{j} / L\right) z, t_{2}-\left(\rho_{1}^{2} / 2 K^{2} L^{2}\right) s\right) d \lambda \\
& +f\left(x_{2}, t_{2}-\left(\rho_{1}^{2} / 2 K^{2} L^{2}\right) s\right)
\end{aligned}
$$

and using (3.2a). Finally $B_{5}$ is estimated as $B_{3}$ was. It follows that there is a constant $C^{\prime}=C^{\prime}(K, L)$ such that

$$
\left|B_{1}\right| \leq C^{\prime}\left[\zeta\left(\left|x_{1}-x_{2}\right|\right)+\zeta\left(\left|t_{1}-t_{2}\right|^{1 / 2}\right)\right] \text {, }
$$

which in conjunction with the estimate for $A_{1}$, implies (3.3a).

To prove (3.3b) and (3.3c), we note that, as in Theorem 1.3, we can obtain the following estimates:

$$
\begin{aligned}
& \left|D_{\imath \jmath} F(x, t, \tau, \sigma)\right| \leq K L \zeta(|\tau| / L) /|\tau| \\
& \left|D_{i \sigma} F(x, t, \tau, \sigma)\right| \leq(1+K) \zeta\left(\sigma^{1 / 2} / K L\right) /|\sigma| \\
& \left|D_{i \tau} F(x, t, \tau, \sigma)\right| \leq(n+K) \zeta(|\tau| / L) /|\tau| \\
& \left|D_{\sigma \tau} F(x, t, \tau, \sigma)\right| \leq(1+K) \zeta\left(\sigma^{1 / 2} / K L\right) /(\sigma L) \\
& \left|D_{\sigma \sigma} F(x, t, \tau, \sigma)\right| \leq(1+K) \zeta\left(\sigma^{1 / 2} / K L\right) /\left(K L \sigma^{3 / 2}\right) \\
& \left|F_{t}(x, t, \tau, \sigma)\right| \leq L K \zeta\left(\sigma^{1 / 2} / K L\right) / \sigma .
\end{aligned}
$$

The desired estimates follow from these inequalities and the method of Theorem 1.3. 
4. Applications. We now describe briefly some applications of the regularized distance.

The first application is an extension of the Hopf boundary point lemma [6]; this extension is similar to results of Kamynin and Khimchenko [12]. Before stating the result, we give some definitions.

Suppose $\Omega$ has a $C^{1}$ local representation $f$ at $x_{0} \in \partial \Omega$. We say that $\Omega$ is Dini at $x_{0}$ if $D f(0)=0$ and if there is a function $\omega$ such that

$\omega$ is increasing and continuous on $[0,8 \delta]$

$$
\begin{aligned}
& \omega(0)=0 \\
& \int_{0}^{8 \delta}(\omega(s) / s) d s \text { is finite }
\end{aligned}
$$

$$
\left|D f\left(x^{\prime}\right)-D f\left(y^{\prime}\right)\right| \leq \omega\left(\left|x^{\prime}-y^{\prime}\right|\right) \text { for }\left|x^{\prime}\right|,\left|y^{\prime}\right|<4 \delta .
$$

We say that $\Omega$ satisfies an interior Dini condition at $x_{0} \in \partial \Omega$ if there is an open set $\Omega^{\prime} \subset \Omega$ such that $x_{0} \in \partial \Omega^{\prime}$ and $\Omega^{\prime}$ is Dini at $x_{0}$. We use the constants $A$ and $\delta$, the function $\omega$, and the coordinate system $Y$ from the various definitions without further comment. We denote by $\nu$ the unit vector in the $y^{n}$ direction and by $d$ the distance to $\partial \Omega^{\prime}$, and we follows the summation convention for repeated indices.

THEOREM 4.1. Let $\mathscr{L}$ be a linear second-order differential operator

$$
\mathscr{L} u=a^{i j} D_{\imath j} u+b^{i} D_{\imath} u+c u
$$

defined on an open set $\Omega \subset \mathbf{R}^{n}$ with the matrix $\left(a^{i j}(x)\right)$ positive semi-definite and symmetric for all $x \in \Omega$. Suppose that $u \in C^{0}(\bar{\Omega}) \cap C^{2}(\Omega)$, that $\mathscr{L} u \leq$ 0 in $\Omega$, that there is a point $x_{0} \in \partial \Omega$ for which $u\left(x_{0}\right) \geq 0$ and $u\left(x_{0}\right) \geq u(x)$ for all $x \in \Omega$ and that $\Omega$ satisfies an interior Dini condition at $x_{0}$. Let $\bar{\omega}$ be a function satisfying (4.1), let $A_{2}$ be a positive constant, set $\omega_{1}(x)=$ $\bar{\omega}(d(x)) / \omega(2 d(x)), \omega_{2}(x)=\bar{\omega}(d(x)) / d(x)$, and suppose that,

$$
\begin{aligned}
& 0<\sum_{i} a^{i i}(x) \leq a^{i j}(x) \nu_{i} \nu_{J} /\left(20 \omega\left(\left|x-x_{0}\right|\right)\right)^{2} \\
& \sum_{i} a^{u l}(x) \leq A_{2} \omega_{1}(x) a^{i j}(x) \nu_{i} \nu_{j} \\
& b(x) \nu_{i} \geq-A_{2} \omega_{2}(x) a^{i j}(x) \nu_{i} \nu_{j} \\
& \sum_{i}\left|b^{i}(x)\right| \leq A_{2}\left[\omega_{2}(x) / \omega\left(\left|x-x_{0}\right|\right)\right] a^{l j}(x) \nu_{i} \nu_{j} \\
& 0 \geq c(x) \geq-A_{2}\left[\omega_{2}(x) / d(x)\right] a^{i j}(x) \nu_{u} \nu_{j}
\end{aligned}
$$

for all $x \in \Omega_{\delta}^{\prime}$. Then for any vector $\mu$ such that $\mu \cdot \nu>0$, we have

$$
\limsup _{t \rightarrow 0}\left(u\left(x_{0}+t \mu\right)-u\left(x_{0}\right)\right) / t<0 .
$$


Proof. By standard arguments, it suffices to find a function $h \in$ $C^{2}\left(\Omega_{\delta}^{\prime}\right) \cap C^{1}\left(\bar{\Omega}_{\delta}^{\prime}\right)$ such that $\mathscr{L} h \geq 0$ in $\Omega_{\delta}^{\prime}, h=0$ on $\partial \Omega_{\delta}^{\prime} \backslash \partial \mathrm{B}_{\delta}, h>0$ on $\Omega_{\delta}^{\prime}, \partial h / \partial y^{n}>0$ at $x_{0}$. We determine $h$ as a function of local regularized distance on $\Omega^{\prime}$, say $h(x)=k(\rho(x))$, and we suppose that $k(0)=0$ and that $k^{\prime}>0$ and $k^{\prime \prime} \geq 0$ on $(0, \delta)$; these properties will be verified from the explicit formula for $k$. By direct calculation,

$$
\mathscr{L} h=k^{\prime \prime} a^{i j} D_{i} \rho D_{j} \rho+k^{\prime}\left(b^{i} D_{i} \rho+a^{i j} D_{i j} \rho\right)+k c
$$

and

$$
\begin{aligned}
a^{i j} D_{i} \rho D_{i} \rho & =a^{i j} \nu_{l} \nu_{j}+a^{i j}\left(D_{i} \rho-\nu_{i}\right) \nu_{j}+a^{i j} D_{i} \rho\left(D_{j} \rho-\nu_{j}\right) \\
& \geq \frac{1}{2} a^{i j} \nu_{\imath} \nu_{j}-\frac{1}{2} a^{i j} D_{\rho} D_{J} \rho-a^{i j}\left(D_{i} \rho-\nu_{\imath}\right)\left(D_{j} \rho-\nu_{j}\right) .
\end{aligned}
$$

Since $D \rho\left(x_{0}\right)=\nu$, we infer from (4.3a) and Theorem 2.1 that

$$
a^{i j} D_{i} \rho D_{j} \rho \geq \frac{1}{6} a^{i j} \nu_{i} \nu_{j}
$$

The other terms in the expression for $\mathscr{L} h$ are estimated similarly via Theorem 2.1, (4.1a), (4.3b-e), and the inequality $k(\rho) \leq \rho k^{\prime}(\rho)$ which follows from $k^{\prime \prime} \geq 0$. Hence for some constant $H=H\left(A, A_{2}, n\right)$ we have

$$
\mathscr{L} h \geq \frac{1}{6} a^{l j} \nu_{i} \nu_{j}\left(k^{\prime \prime}-H k^{\prime} \bar{\omega}(2 \rho) / \rho\right) .
$$

Taking (4.1c) into account, we see that the desired function is

$$
k(\rho)=\int_{0}^{\rho} \exp \left(H \int_{0}^{r} \bar{\omega}(2 s) / s d s\right) d r .
$$

It is instructive to compare this theorem with [12, Theorem 1] and similar results of Kamynin and Khimchenko. The proof of our Theorem 3.1 is simple and our method is well-suited to conditions stated purely in terms of the distance function $d$. On the other hand, Kamynin and Khimchenko consider a relaxation of our (4.3b). In addition they are able to prove more easily the sharpness of the Dini condition (4.1c) for this result and those discussed below.

Of course an analogous boundary part lemma for parabolic equations can be proved via Theorem 3.1 (cf. [9], [13]). We mention also [21] in which a boundary-point-type lemma is proved for Lipschitz domains. All of these boundary point results lead to uniqueness theorems for suitable boundary value problems.

Another application of the regularized distance occurs in the study of regularity at the boundary for solutions of boundary vlaue problems. For linear equations we refer to [10] and [11], and we only remark that the 
results there can be obtained via regularized distance. For nonlinear equations, we refer to [18] and [19] although we remark that some of the results thee concerning convex domains can be improved by using Theorem 1.4. For example [18, Theorem A.1] can be proved directly by the methods of [18, Chapter II] since a global regularized distance is available. Similarly the hypothesis that $\partial \Omega \in C^{2}$ can be removed from [10, Theorems 3.3 and 3.9]. In particular we have the following result: if $\Omega$ is bounded and convex, if $u \in C^{0}(\bar{\Omega}) \cap C^{2}(\Omega)$ is a solution of the minimal surface equation

$$
\mathscr{M} u=\left(1+|D u|^{2}\right) \Delta u-D_{i} u D_{j} u D_{i j} u=0
$$

in $\Omega$, and if $\left.u\right|_{\partial \Omega}$ is Lipschitz, then $u$ is Hölder continuous on $\bar{\Omega}$. In connection with these results on nonlinear equations, we raise an important question concerning the regularized distance: Is there a natural analog of Serrin's curvature conditions [23] for domains which are less smooth than $C^{2}$ ? Since in the theory of elliptic equations, these conditions are used to infer estimates on the Hessian matrix $\left(D_{i j} d\right)$ of the distance function, it seems reasonable to assume that the analog exists. So far, however, we have not been able to find a simple geometric condition which implies the appropriate behavior for the Hessian $\left(D_{i j} \rho\right)$ of the regularized distance.

As a final application, we discuss extensions of functions on $\partial \Omega$ to globally defined functions. In what follows, we assume $\Omega$ to be bounded although an unbounded $\Omega$ can also be handled by our methods. Our first step is to define appropriate regularity classes of functions in terms of certain norms. We denote by $\zeta$ a continuous increasing function with $\zeta(0)=0$ and $\zeta(t)>0$ for $t>0$ such that $\zeta(t) / t$ is a decreasing function of $t$. (From [20, §3.5] it follows that assuming this last property involves no loss of generality for our purposes), and we get $\mathrm{Z}(t)=\log _{t} \zeta(t)$ ( $\mathrm{Z}=$ capital $\zeta$ ). For $u$ defined on $\Omega$ and $k \geq 0$ an integer, we define

$$
\begin{aligned}
|u|_{0} & =\sup _{\Omega} u, \quad\|u\|_{k}=\sum_{j=0}^{k}\left|D^{j} u\right|_{0} \\
{[u]_{z} } & =\sup \{|u(x)-u(y)| / \zeta(|x-y|): x \neq y \text { in } \Omega\} \\
|u|_{k+Z} & =\|u\|_{k}+\left[D^{k} u\right]_{z} .
\end{aligned}
$$

We note that $|u|_{k+z}$ is the usual Hölder norm ||$_{k+\alpha}$ when $\zeta(t)=t^{\alpha}$ for some $0<\alpha \leq 1$. We also note that $\left\|_{0}=\right\| \|_{0}$ and that \|\|$_{k} \geq\|\|_{k}$ for any integer $k$. We denote by $H_{k+Z}(\Omega)$ the set of all $u$ for which $|u|_{k+Z}$ is finite. Let $\gamma$ be a positive function defined on $\{t>0\}$ with the property that 
there are positive constants $\gamma_{1}$ and $\gamma_{2}$ such that

$$
\gamma_{1} \gamma(2 t) \leq \gamma(t) \leq \gamma_{2} \gamma(2 t) \text { for all } t>0 \text {. }
$$

For $\delta>0$, we write $\Omega_{\delta}=\{x \in \Omega: d(x)>\delta\}$, and for $a \geq 0$ and $k$ a non-negative integer such that $t^{a} \gamma(t)$ and $t^{k} \gamma(t)$ are increasing functions of $t$, we define the norms

$$
\begin{gathered}
|u|_{a}^{(\Gamma)}=\sup _{\delta>0}\left\{\delta^{a} \gamma(\delta)|u|_{a ; \Omega_{\delta}}\right\} \\
\|u\|_{k}^{(\Gamma)}=\sup _{\delta>0}\left\{\delta^{a} \gamma(\delta)\|u\|_{k ; \Omega_{\delta}}\right\} .
\end{gathered}
$$

In analogy with the weighted Hölder spaces of [3] we denote by $H_{a}^{(\Gamma)}$ and $C_{k}^{(\Gamma)}$ the set of all functions $u$ on $\Omega$ with finite norm $|u|_{a}^{(\Gamma)}$ and $\|u\|_{k}^{(\Gamma)}$ respectively. Thus (1.7) can be reformulated as saying $\rho \in C_{2}^{(-1-Z)}$. In fact a stronger result is valid.

THEOREM 4.1. Under the hypotheses of Theorem 1.3 (including (1.6)) $\Omega$ has a regularized distance $\rho \in H_{a}^{(-1-Z)}$ for any $a \geq 2$.

Proof. As in the proof of Theorem 1.3, we see that $\rho \in C_{k}^{(-1-Z)}$ for all integers $k>2$. We first show that $H_{2+\alpha}^{(-1-Z)} \supset C_{2}^{(-1-Z)} \cap C_{3}^{(-1-Z)}$ for all $\alpha \in(0,1]$, and then that $H_{2}^{(-1-Z)} \supset H_{1+Z} \cap C_{2}^{(-1-Z)}$, thus establishing the theorem for $2 \leq a \leq 3$. The general case $a>3$ is handled similarly.

Let $u \in C_{2}^{(-1-Z)} \cap C_{3}^{(-1-Z)}$. Setting

$$
\begin{aligned}
H & =\sup _{\delta>0}\left\{\left(\delta^{1+\alpha} / \zeta(\delta)\right)\left[D^{2} u\right]_{\alpha ; \Omega_{\delta}}\right\}, \\
d^{*} & =\max \{1, \operatorname{diam} \Omega\},
\end{aligned}
$$

we see by some simple algebra that

$$
|u|_{2+\alpha}^{(-1-Z)} \leq H+\left(d^{*}\right)^{\alpha}\|u\|_{2}^{(-1-Z)} \text {. }
$$

Hence we need only estimate $H$ in terms of $c_{1}=\left|D^{3} u\right|_{0}^{(2-Z)}$ and $c_{2}=$ $\left|D^{2} u\right|_{0}^{(1-\mathrm{Z})}$ since $c_{1} \leq\|u\|_{3}^{(-1-\mathrm{Z})}$ and $c_{2} \leq\|u\|_{2}^{(-1-\mathrm{Z})}$. Now fix $\delta>0$, positive integers $i \leq n$ and $j \leq n$, and $x$ and $y$ in $\Omega_{\delta}$. If $|x-y|<\delta / 2$, then $x+t(y-x) \in \Omega_{\delta / 2}$ for $0 \leq t \leq 1$, so

$$
\begin{aligned}
\left|D_{\imath \jmath} u(x)-D_{\imath j} u(y)\right| & =\left|\int_{0}^{1} D_{i j k} u(x+t(y-x)) d t\left(x_{k}-y_{k}\right)\right| \\
& \leq 4 c_{1}|x-y| \zeta(\delta / 2) \delta^{-2} \leq 4 c_{1}|x-y|^{\alpha} \zeta(\delta) \delta^{-1-\alpha} .
\end{aligned}
$$

On the other hand if $|x-y| \geq \delta / 2$, then

$$
\begin{aligned}
\left|D_{\imath j} u(x)-D_{i j} u(y)\right| & \leq\left|D_{\imath \jmath} u(x)\right|+\left|D_{\imath j} u(y)\right| \\
& \leq 2 c_{2} \zeta(\delta) \delta^{-1} \leq 4 c_{2}|x-y|^{\alpha} \zeta(\delta) \delta^{-1-\alpha} .
\end{aligned}
$$


Hence $H \leq 4 n\left(c_{1}+c_{2}\right)$, yielding $H_{2+\alpha}^{(-1-\mathrm{Z})} \supset C_{2}^{(-1-\mathrm{Z})} \cap C_{3}^{(-1-\mathrm{Z})}$.

To show that $H_{2}^{(-1-Z)} \supset C_{2}^{(-1-Z)} \cap H_{1+Z}$, we proceed as before except that in the case $|x-y| \geq \delta / 2$, we use the estimate

$$
\begin{aligned}
|D u(x)-D u(y)| & \leq \zeta(|x-y|)=(\zeta(|x-y|) /|x-y|)|x-y| \\
& \leq 2|x-y| \zeta(\delta) / \delta .
\end{aligned}
$$

It should be noted that the inclusions $H_{2+\alpha}^{(-1-Z)} \supset C_{2}^{(-1-Z)} \cap C_{3}^{(-1-Z)}$ and $H_{2}^{(-1-Z)} \supset H_{1+Z} \cap C_{2}^{(-1-Z)}$ were established without any restrictions on the smoothness of $\partial \Omega$. Under slightly stronger hypotheses (e.g., $\partial \Omega$ is Lipschitz and $\mathrm{Z}=\beta$, a constant, with $0<\beta<1$ ), better results are valid; see [3, Lemma 2.1].

We are now ready to discuss extensions of functions on $\partial \Omega$. For $k$ and $\zeta$ as above, we write $H_{k+Z}^{\prime}$ for the set of all $h \in H_{k+Z}\left(\mathbf{R}^{n}\right)$ with compact support and we write $H_{k+Z}(\partial \Omega)$ for the set of all $h$ with finite norm:

$$
|h|_{k+Z ; \partial \Omega}=\inf \left\{|\tilde{h}|_{k+Z}: \tilde{h} \in H_{k+Z}^{\prime} \text { and } \tilde{h}=h \text { on } \partial \Omega\right\} .
$$

We note that if $h$ is continuous on $\partial \Omega$, then $h \in H_{Z}(\partial \Omega)$ for some $\zeta$. Moreover if $\partial \Omega$ has a local representation at each $x_{0} \in \partial \Omega$ and if $\mid h(x)-$ $h(y) \mid \leq \zeta(|x-y|)$ for all $x$ and $y$ in $\partial \Omega$ and some $\zeta$, then $h \in H_{Z}(\partial \Omega)$ and $|h|_{z ; \partial \Omega}=1$. If, in addition, all the local representations $f$ are in $C^{k}$ for some $k \geq 1$ then $H_{k+Z}(\partial \Omega)$ (for suitable $\zeta$ ) and $H_{j+Z}(\partial \Omega$ ) (for all $j<k$ and all $\zeta)$ can be defined via the function $g$ given by $g\left(y^{\prime}\right)=h\left(y^{\prime}, f\left(y^{\prime}\right)\right)$ being in the appropriate function space. For brevity we write $\partial \Omega \in H_{k+Z}$ if $\partial \Omega$ has a local $H_{k+Z}$ represenentation at each $x_{0} \in \partial \Omega$. In this case we can extend functions on $\partial \Omega$ in a convenient fashion (cf. [5, Lemma 1]).

THEOREM 4.2. (a) If $h_{0} \in H_{\mathrm{Z}}(\partial \Omega)$, then there is $\tilde{h} \in H_{Z}^{\prime}$ such that $\tilde{h}=h_{0}$ on $\partial \Omega$ and $|\tilde{h}|_{a}^{(-Z)} \leq c(a, \Omega)|h|_{Z ; \partial \Omega}$ for all $a \geq 1$. (b) Suppose $\partial \Omega \in H_{1+Z}$ and let $\nu$ be the inner normal to $\partial \Omega$. If $h_{0} \in H_{1+Z}(\partial \Omega)$ and if $h_{1} \in H_{Z}(\partial \Omega)$ then there is $\tilde{h} \in H_{1+Z}^{\prime}$ such that

$$
\tilde{h}=h_{0}, \quad D \tilde{h} \cdot \nu=h_{1} \quad \text { on } \partial \Omega
$$

and

$$
|h|_{a}^{(-1-Z)} \leq c(a, \Omega)\left(|h|_{1+Z ; \partial \Omega}+\left|h_{1}\right|_{Z ; \partial \Omega}\right)
$$

for all $a \geq 2$.

Proof. Set $H(x, \tau)=\int_{|z|<1} h_{0}(x-(\tau / L) z) \phi(z) d z$ with $\phi$ as in $\S 1$.

(a) $\tilde{h}(x)=H(x, \rho(x))$ gives the desired function. 
(b) Note that according to (a), $1 / D \rho \cdot \nu$ can be extended to a function $F \in H_{Z}^{\prime} \cap H_{a}^{(-Z)}$. Using the obvious definition for $H_{1}(x, \tau)$, we set $h_{2}(x)$ $=H(x, \rho(x))$ and $h_{3}(x)=H_{1}(x, \rho(x))$. Then

$$
\tilde{h}(x)=h_{2}(x)+\left(h_{3}(x)-D h_{2}(x) \cdot D \rho(x)\right) F(x) \rho(x)
$$

gives the desired function.

Acknowledgments. I would like to thank Professors David Gilbarg, Elgin Johnston, Andrejs Treibergs, Roger Alexander, and A. M. Fink for many useful conversations. I would also like to thank the referee of an earlier version of this paper for drawing my attention to the work of Kamynin and Khimchenko, and that of Výborný.

\section{REFERENCES}

[1] W. H. Fleming, Functions of Several Variables, 2nd ed., Springer-Verlag, Berlin, 1977.

[2] L. E. Fraenkel, On the regualarity of the boundary in the theory of Sobolev spaces, Proc. London Math. Soc., (3) 39 (1979), 385-427.

[3] D. Gilbarg and L. Hörmander, Intermediate Schauder estimates, Arch. Rational Mech. Anal., 74 (1980), 297-318.

[4] D. Gilbarg and N. S. Trudinger, Elliptic Partial Differential Equations of Second Order, 2nd ed., Springer-Verlag, Berlin, 1983.

[5] E. Giusti, Boundary behavior of non-parametric minimal surfaces, Indiana Univ. Math. J., 22 (1972), 435-444.

[6] E. Hopf, A remark on linear elliptic differential equations of second order, Proc. Amer. Math. Soc., 3 (1952), 791-793.

[7] L. I. Kamynin, Smoothness of heat potentials. I. Heat potentials on a surface of type $\Lambda_{1, \alpha, \alpha / 2}^{0,1,(1+\alpha) / 2}$, Differential Equations, 1 (1965), 613-647.

[8] L. I. Kamynin and B. N. Khimchenko, The maximum principle for an elliptic-parabolic equation of the second order, Siberian Math. J., 13 (1972), 533-545.

[9] Analogs of Giraud's theorem for a second-order parabolic equation, Siberian Math. J., 14 (1973), 59-66.

[10] - The principle of the maximum and boundary Lipschitz bounds for the solution of a second order elliptic parabolic equation, Siberian Math. J., 15 (1974), 242-260.

[11] , The maximum principle and local Lipschitz estimates near the lateral boundary for solutions of second order parabolic equations, Siberian Math. J., 16 (1975), 897-909.

[12] , Development of Aleksandrov's theory of the isotropic strict extremum principle, Differential Equations, 16 (1980), 181-189.

[13] Theorems of Giraud type for second order parabolic equations with admit degeneration, Siberian Math. J., 21 (1981), 535-551.

[14] S. G. Krantz and H. R. Parks, Distance to $C^{k}$ hypersurfaces, J. Differential Equations, 40 (1981), 116-120.

[15] A. Kufner, Weighted Sobolev Spaces, Teubner-Texte, Leipzig, 1980.

[16] O. A. Ladyženskaja, V. A. Solonnikov, and N. N. Ural'ceva, Linear and Quasilinear Equations of Parabolic Type, Amer. Math. Soc., Providence, R. I., 1968.

[17] S. R. Lay, Convex Sets and Their Applications, Wiley-Interscience, New York, 1982.

[18] G. M. Lieberman, The quasilinear Dirichlet problem with decreased regularity at the boundary, Comm. Partial Differential Equations, 6 (1981), 437-497. 
[19] The Dirichlet problem for quasilinear elliptic equations with Hölder continuous boundary values, Arch. Rational Mech. Anal., 79 (1982), 305-323.

[20] G. G. Lorentz, Approximation of Functions, Holt, Reinhart, and Winston, New York, 1966.

[21] N. S. Nadirašvili, A lemma on the inner derivative and the uniqueness of the solution of the second boundary value problem for second order elliptic equations, Soviet Math. Doklady, 24 (1981), 598-601.

[22] J. Nečas, Sur une méthode pour resondre les équations aux dérivies partielles du type elliptique, voisine de la variationelle, Ann. Scuola Norm. Sup. Pisa, (3) 16 (1962), 305-326.

[23] J. Serrin, The problem of Dirichlet for quasilinear equations with many independent variables, Philos. Trans. Roy. Soc. London, Ser. A, 264 (1969), 413-496.

[24] H. Triebel, Interpolation Theory, Function Spaces, Differential Operators, North-Holland, New York, Oxford, 1978.

[25] R. Výborný, On certain extensions of the maximum principle, in Differential Equations and their Applications (Proc. Conf. Prague, 1962) Publ. House Czeckoslavak Acad. Sci., Prague; Academic Press, New York, 1963: 223-228.

Received March 10, 1983.

IOWA STATE UNIVERSITY

AMES, IA 50011 


\section{PACIFIC JOURNAL OF MATHEMATICS EDITORS}

DONALD BABBITT (Managing Editor)
University of California
Los Angeles, CA 90024
CHARLES R. DEPrIMA
California Institute of Technology
Pasadena, CA 91125
R. FINN
Stanford University
Stanford, CA 94305

\author{
HERMANN FLASCHKa \\ University of Arizona \\ Tucson, AZ 85721 \\ RAMESH A. GANGOLLI \\ University of Washington \\ Seattle, WA 98195 \\ ROBION KIRBY \\ University of California \\ Berkeley, CA 94720 \\ C. C. Moore \\ University of California \\ Berkeley, CA 94720
}

Hugo Rossi

University of Utah

Salt Lake City, UT 84112

H. SAMELSON
Stanford University
Stanford, CA 94305

HAROLD STARK

University of California, San Diego

La Jolla, CA 92093

\section{ASSOCIATE EDITORS}
R. ARens
E. F. BECKENBACH (1906-1982)
B. H. NeUmanN
F. WOLF
K. YOSHIDA

\begin{tabular}{ll}
\multicolumn{2}{c}{ SUPPORTING INSTITUTIONS } \\
UNIVERSITY OF ARIZONA & UNIVERSITY OF OREGON \\
UNIVERSITY OF BRITISH COLUMBIA & UNIVERSITY OF SOUTHERN CALIFORNIA \\
CALIFORNIA INSTITUTE OF TECHNOLOGY & STANFORD UNIVERSITY \\
UNIVERSITY OF CALIFORNIA & UNIVERSITY OF HAWAII \\
MONTANA STATE UNIVERSITY & UNIVERSITY OF TOKYO \\
UNIVERSITY OF NEVADA, RENO & UNIVERSITY OF UTAH \\
NEW MEXICO STATE UNIVERSITY & WASHINGTON STATE UNIVERSITY \\
OREGON STATE UNIVERSITY & UNIVERSITY OF WASHINGTON
\end{tabular}

The Supporting Institutions listed above contribute to the cost of publication of this Journal, but they are not owners or publishers and have no responsibility for its content or policies.

\footnotetext{
Mathematical papers intended for publication in the Pacıfic Journal of Mathematıcs should be in typed form or offset-reproduced (not dittoed), double spaced with large margins. Please do not use built up fractions in the text of the manuscript. However, you may use them in the displayed equations. Underline Greek letters in red, German in green, and script in blue. The first paragraph must be capable of being used separately as a synopsis of the entire paper. In particular it should contain no bibliographic references. Please propose a heading for the odd numbered pages of less than 35 characters. Manuscripts, in triplicate, may be sent to any one of the editors. Please classify according to the scheme of Math. Rev ews, Index to Vol. 39. Supply name and address of author to whom proofs should be sent. All other communications should be addressed to the managing editor, or Elaine Barth, University of California, Los Angeles, California 90024.

There are page-charges associated with articles appearing in the Pacific Journal of Mathematics. These charges are expected to be paid by the author's University, Government Agency or Company. If the author or authors do not have access to such Institutional support these charges are waived. Single authors will receive 50 free reprints; joint authors will receive a total of 100 free reprints. Additional copies may be obtained at cost in multiples of 50 .
}

The Pacific Journal of Mathematics is issued monthly as of January 1966. Regular subscription rate: $\$ 190.00$ a year (5 Vols., 10 issues). Special rate: $\$ 66.00$ a year to individual members of supporting institutions.

Subscriptions, orders for numbers issued in the last three calendar years, and changes of address should be sent to Pacific Journal of Mathematics, P.O. Box 969, Carmel Valley, CA 93924, U.S.A. Old back numbers obtainable from Kraus Periodicals Co., Route 100, Millwood, NY 10546.

The Pacific Journal of Mathematics at P.O. Box 969, Carmel Valley, CA 93924 (ISSN 0030-8730) publishes 5 volumes per year. Application to mail at Second-class postage rates is pending at Carmel Valley, California, and additional mailing offices. Postmaster: Send address changes to Pacific Journal of Mathematics, P.O. Box 969, Carmel Valley, CA 93924.

PUBLISHED BY PACIFIC JOURNAL OF MATHEMATICS, A NON-PROFIT CORPORATION Copyright $\odot 1985$ by Pacific Journal of Mathematics 


\section{Pacific Journal of Mathematics}

\section{Vol. 117, No. 2 \\ February, 1985}

Robert Walter Bagley, Ta-Sun Wu and J. S. Yang, On a class of topological groups more general than SIN groups ............... 209

Bruce Alan Barnes, Algebraic elements of a Banach algebra modulo an ideal ...............................................219

Howard D. Fegan and Peter Gilkey, Invariants of the heat equation .......233

Erica Flapan, Necessary and sufficient conditions for certain homology

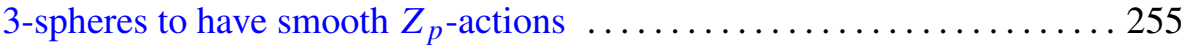

Philip R. Heath, Product formulae for Nielsen numbers of fibre maps . . . . 267

Derbiau Frank Hsu and A. Donald Keedwell, Generalized complete mappings, neofields, sequenceable groups and block designs. II ...... 291

Taqdir Husain, Orthogonal primitive idempotents and Banach algebras

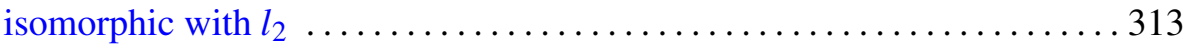

Gary M. Lieberman, Regularized distance and its applications . . . . . . . 329

William W. Menasco, Determining incompressibility of surfaces in alternating knot and link complements ............................ 353

Benjamin Muckenhoupt, Weighted reverse weak type inequalities for the Hardy-Littlewood maximal function $\ldots \ldots \ldots \ldots \ldots \ldots \ldots \ldots \ldots \ldots \ldots \ldots$

John Dacey O'Neill, Direct summands of direct products of slender

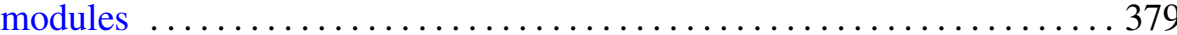

Andreas Rapp, Elimination of Malitz quantifiers in stable theories 387

Francisco José Ruiz, A unified approach to Carleson measures and $A_{p}$ weights

Hanamatagouda Pandappa Sankappanavar, Heyting algebras with dual

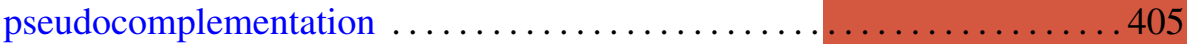

Annette Sinclair, Strong Carleman and strong uniform approximation . . . . 417

Masaaki Suzuki, The generalized Schwarz lemma for the Bergman metric

Brian Thorpe and Ludwig Tomm, Universal approximation by regular

weighted means 